\title{
Chirurgische Therapieoptionen bei obstruktiven schlafbezogenen Atmungsstörungen und Schnarchen
}

Maren Just, Andreas Dietz

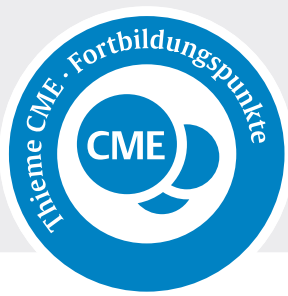

Entsprechend der ICSD-3 wird eine obstruktive Schlafapnoe (OSA) dann diagnostiziert, wenn die Atmungsstörung durch keine andere Schlafstörung oder medizinische Erkrankung oder durch Medikamente oder andere Substanzen erklärbar ist und entweder ein AHI (Apnoe-Hypopnoe-Index-Anzahl der Apnoen und Hypopnoen pro Stunde Schlaf) > 15/h (Ereignis $\geq 10$ s) Schlafzeit oder ein AHI $\geq 5 / h$ Schlafzeit in Kombination mit einer typischen klinischen Symptomatik oder relevanten Komorbidität vorliegt [1].

\section{Einleitung}

Schlafbezogene Atmungsstörungen (SBAS) treten ausschließlich oder überwiegend im Schlaf auf. Sie wirken sich störend auf den Schlaf aus und beeinträchtigen die Erholungsfunktion erheblich. Bei Kindern ist „die herausragende Lernfähigkeit mit einer Menge an nächtlichem Tiefschlaf verknüpft“ [2]. Bei Erwachsenen erhöht schlechter, nicht erholsamer Schlaf die Tagesschläfrigkeit, führt zu vermehrter Unfallneigung und ist ein häufiger Risikofaktor für kardiovaskuläre Erkrankungen. Im Vergleich zu Gesunden zeigen Schlafapnoeiker ein erhöhtes Morbiditäts- und Mortalitätsrisiko. 20\% der Erwachsenen sind von einer milden, ca. 6-7\% von einer mittel- bis hochgradigen OSA betroffen. Die Prävalenz des reinen Schnarchens ist altersabhängig und liegt weitaus höher: bei ca. $60 \%$ bei älteren Männern und 50 \% bei postmenopausalen Frauen.

\section{Merke}

Die klinische Bedeutung der OSA liegt in den langfristigen Folgen für das kardiovaskuläre System sowie in den Folgen des nicht erholsamen Schlafes mit erhöhter Tagesschläfrigkeit und Vigilanzminderung:

- Erhöhtes Risiko für Hypertonie, Herzinsuffizienz, Schlaganfall

- 3-7-fach erhöhtes Unfallrisiko im Straßenverkehr

Durch die Entwicklung von internationalen Standards in der Diagnostik und Therapie von Schlafstörungen hat die Schlafmedizin einen festen Platz in der medizinischen Versorgung eingenommen. Für die richtige Therapieentscheidung sind Kenntnisse und Grundbegriffe der Schlafmedizin und ihrer Bewertung unabdingbar. Obstruktionen im Bereich der oberen Luftwege zählen zu den häufigsten Ursachen schlafbezogener Atmungsstörungen und liegen damit diagnostisch und therapeutisch im Fokus des HalsNasen-Ohrenarztes. Das Erkennen der obstruktiven Ursa- chen sowie die Abgrenzung obstruktiver Faktoren gegenüber anderen schlafbezogenen Atmungsstörungen ist von essentieller Bedeutung und bedarf in der Regel einer interdisziplinären Abstimmung - insbesondere mit Schlafmedizinern, Pneumologen, Kinderärzten und Kardiologen [4].

HNO-Ärzte können nach Anerkennung ihres Facharztes die Zusatzbezeichnung „Schlafmedizin“ erwerben. Für weitere Informationen wird auf die zuständigen Landesärztekammern verwiesen.

\footnotetext{
Merke

Schlafbezogene Atmungsstörungen (SBAS)

Ausschließlich oder primär im Schlaf auftretende Atmungsstörung charakterisiert durch:

- Apnoen oder Hypopnoen mit oder ohne pharyngealer Obstruktion

- Hypoventilationen

- Hypoxämie, Hyperkapnie, Azidose
}

\section{Schnarchen und obstruktive Schlafapnoe}

Aktuell existiert noch keine zufriedenstellende Definition des Schnarchens (Rhonchopathie). Das Schnarchen, als eigenständiges Phänomen, beschreibt inspiratorische akustische Atemgeräusche. Diese gehen per se weder mit einer Schlafstörung noch mit einer gesteigerten Schläfrigkeit oder einem erhöhten kardiovaskulären Risiko einher. Es entsteht durch die Abnahme des Muskeltonus im Bereich der oberen Atemwege mit Erhöhung der Vibrationsbereitschaft von Weichteilstrukturen des oberen Luftwegs, insbesondere des Weichgaumens [5]. Reines Schnarchen ist für den Betroffenen folgenlos, kann allerdings die Ursache sozialer Probleme darstellen. Die Diagnose des harmlosen 
- Tab. 1 Schweregradeinteilung der obstruktiven Schlafapnoe (OSA) nach dem Apnoe-Hypopnoe-Index (AHI).

\begin{tabular}{|l|l|}
\hline Primäres Schnarchen & $\mathrm{AHI}<5$ \\
\hline Leichtgradige OSA & $\mathrm{AHI}<15$ \\
\hline Mittelgradige OSA & $\mathrm{AHI}>15-30$ \\
\hline Schwergradige OSA & $\mathrm{AHI}>30$ \\
\hline AHI, Apnoe-Hypopnoe-Index & \\
\hline
\end{tabular}

Schnarchens lässt sich erst nach Ausschluss einer schlafbezogenen Atmungsstörung diagnostizieren. Intensives, arrhythmisches Schnarchen kann ein Hinweis für obstruktive Schlafapnoe sein, weshalb eine schlafmedizinische Differenzialdiagnostik erfolgen sollte. Der Übergang in eine obstruktive Schlafapnoe ist fließend. Gekennzeichnet ist dies durch wiederholte Obstruktionen im oberen Atemweg während des Schlafes und daraus folgenden Apnoen und Hypopnoen mit und ohne Weckreaktionen (Arousals). Während unter Apnoe ein Atemstillstand verstanden wird, ist eine Hypopnoe durch eine Reduktion des Atemflusses gekennzeichnet. Beide Begriffe können anhand apparativer Schlafdiagnostik genauer beschrieben werden. Zur Quantifizierung des Schweregrades dient in erster Linie der Apnoe-Hypopnoe-Index (AHI; - Tab. 1). Bei Kindern gelten bereits mehr als 2 Atempausen pro Stunde Schlaf $(\mathrm{AHI}>2)$ als pathologisch [3][4]. Bezüglich der aktuellen Aspekte in der Diagnostik und nicht operativen Therapie wird auf die entsprechende Literatur verwiesen.

In diesem Artikel soll für den Facharzt für Hals-Nasen-Ohrenheilkunde ein relevanter Überblick über die chirurgischen Therapiemöglichkeiten bei schlafbezogenen Atmungsstörungen gegeben werden.

\section{FALLBEISPIEL}

Ein 37-jähriger Mann (BMI: $27 \mathrm{~kg} / \mathrm{m}^{2}$ ) stellt sich mit zunehmendem Schnarchen und merkbarer Tagesschläfrigkeit und Morgenmüdigkeit vor. Auch die Konzentration sei herabgesetzt. Auf Nachfragen berichtet er über einen nicht erholsamen Schlaf, obwohl er abends gegen 23:00 Uhr ins Bett gehe und morgens um 08:00 Uhr aufstehe. Seine Lebensgefährtin berichtet über lautes Schnarchen und längere Atempausen, welche sie sehr beunruhigen. Er schnarche in jeder Körperlage, bekomme subjektiv gut Luft durch die Nase, rauche nicht und trinke abends gelegentlich ein Bier. Nach ausführlicher Anamnese, klinischer Untersuchung, Abklärung von Begleiterkrankungen und erfolgter ambulanter Polygrafie wurde ein mittelgradiges Schlafapnoesyndrom diagnostiziert. Eine Vorstellung im Schlaflabor wurde primär abgelehnt und nach weiteren diagnostischen Möglichkeiten gefragt.

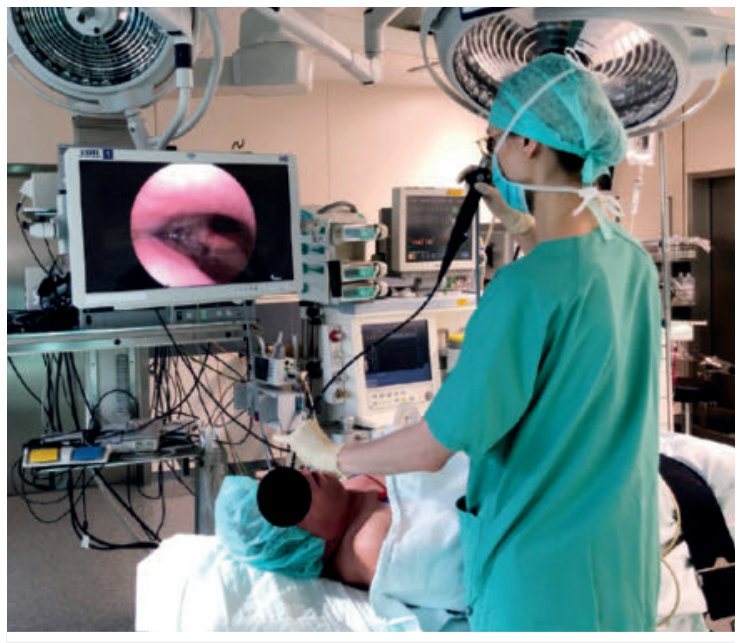

- Abb. 1 Setting einer Schlafendoskopie im Operationssaal.

Der Goldstandard der Therapie der OSA ist die nächtliche Überdruckbeatmung mittels continuous positive airway pressure (CPAP). Die Effektivität dieser Therapie kann jedoch durch eingeschränkte Akzeptanz und Toleranz sowie reduzierte Compliance limitiert sein [6][7][8]. Alternativen können zum Beispiel die Anwendung von intraoralen Schienen und die chirurgische Behandlung darstellen. Chirurgische Behandlungen haben den Vorteil einer 100 \%igen Adhärenz, welche maßgebend die Wirksamkeit einer Therapie beeinflusst [8][9]. Trotz allem ist die chirurgische Therapie nicht die Therapie der ersten Wahl, unter anderem wegen schlechter Resultate bei nicht selektionierten Patienten. Die genaue Beobachtung und Beschreibung der relevanten obstruktiven Areale im oberen Atemweg sind von essentieller Bedeutung. Diese führen zu einer besseren Selektion von Patienten, woraus zufriedenstellende chirurgische Ergebnisse resultieren [10].

\section{Präoperative Diagnostik mittels Schlafendoskopie}

Die alleinige Begutachtung der oberen Atemwege bei wachen Patienten ist nur von begrenzter Aussagekraft. Sie erlaubt die Beschreibung der jeweiligen Anatomie. Rückschlüsse auf Veränderungen im Schlaf können dadurch nicht sicher gegeben werden. Aufgrund des nachlassenden Muskeltonus während des Schlafes können folgende Parameter signifikant anders ausfallen als im Wachzustand:

- Kollapsort

- Kollapsmuster

- Kollapsgrad

Diese Veränderungen der oberen Atemwege können prinzipiell mit der Schlafendoskopie untersucht und dokumentiert werden ( $\triangleright$ Abb. 1). Borowiecki versuchte bereits 1978, die ersten Schlafendoskopien während des physiologischen Schlafes durchzuführen. Dies war aufgrund des 
- Tab. 2 Indikationen und Kontraindikationen der Schlafendoskopie.

\begin{tabular}{|l|l|l|}
\hline Indikation & Kontraindikation & $\begin{array}{l}\text { Relative } \\
\text { Kontraindikation }\end{array}$ \\
\hline $\begin{array}{l}\text { Schnarchen und OSA, ohne } \\
\text { primäre CPAP-Indikation }\end{array}$ & $\begin{array}{l}\text { Schwer reduzierter All- } \\
\text { gemeinzustand (ASA 4) }\end{array}$ & Morbide Adipositas \\
\hline $\begin{array}{l}\text { Therapieversager nach } \\
\text { operativer Primärtherapie } \\
\text { bei OSA }\end{array}$ & Schwangerschaft & \\
\hline $\begin{array}{l}\text { CPAP-Intoleranz/ } \\
\text { CPAP-Versagen }\end{array}$ & $\begin{array}{l}\text { Allergie auf Propofol/ } \\
\text { Narkotika }\end{array}$ & \\
\hline
\end{tabular}

Eignung für Hypoglossus-Stimulation

OSA- Obstruktive Schlafapnoe; ASA- American Society of Anesthesiologists/Scoring-System zur Einteilung von Patienten bezüglich ihres körperlichen Zustandes; CPAP- continuous positive airway pressure.

personellen und zeitlichen Aufwands nicht alltagstauglich. Croft und Pringle nahmen 1991 erste schlafendoskopische Versuche unter Sedierung mit Midazolam vor. Vorteilhaft erwies sich die Sedation in Bezug auf Durchführbarkeit (tagsüber) und besserer Toleranz/Verträglichkeit des nasal eingeführten Endoskops [11]. Ziele des ersten Positionspapiers zur drug-induced sleep endoscopy (DISE) 2014 waren das Vorgehen zu standardisieren, Einsicht in die Hauptaspekte der Durchführung und Auswertung zu geben, um eine Basis für die Vergleichbarkeit und weitere Forschungen zu ermöglichen. Seitdem wurden jedoch zahlreiche neue Studien zur Durchführung, Anwendung neuer Sedativa, Sedationstiefe und Kollapsmuster veröffentlicht, sodass ein Update des Positionspapiers 2017 erfolgte. Konsens fand sich bezüglich der Indikationen und Kontraindikationen ( $\vee$ Tab. 2 ). Die DISE bietet zusätzliche Informationen über die potenziellen Kollapsorte und -muster. Sie kann somit bei ausgewählten Patienten ergänzend zur Beurteilung des oberen Atemwegs und der Therapiefindung eingesetzt werden.

Konsens fand sich auch bezüglich der klinischen Voruntersuchungen, der Patientenselektion, des Durchführungsorts, der technischen Ausstattung, der Durchführung der DISE, der Anwendung von lokalem endonasalem Anästhetikum, der Sedativa, der Patientenpositionierung während der DISE und dem Beobachtungszeitraum.

\section{Beobachtung und Beschreibung der Schlafendoskopie}

Die DISE sollte auf Video aufgezeichnet werden. Dies ist zur Illustration im Patientengespräch und für Vergleiche bei potenziellem Therapieversagen sinnvoll. Pro Atemwegssegment sollten mindestens 2 vollständige Zyklen in der optimalen Sedationstiefe durchgeführt werden. Ein vollständiger Zyklus besteht aus der Abfolge von Schnar- chen, Atemwegsverschluss über die Entsättigung bis zur erneuten Atemwegsöffnung. Beobachtet werden das Kollapsmuster und der Kollapsgrad auf den verschiedenen $\mathrm{Ni}$ veaus des oberen Atemweges (Kollapsorte) [12].

Ebenso fand sich Übereinstimmung bezüglich bestehender Kollapsmuster (antero-posterior, lateral und konzentrisch) als auch der Kollapsorte: antero-posterior oder konzentrische Obstruktion des Weichgaumens, Kollaps der lateralen Pharynxwände, Kollaps auf Zungengrundebene, Epiglottisansaugphänomen, sekundärer epiglottischer Kollaps durch zum Beispiel anatomische Varianten als auch die Beteiligung der aryepiglottischen Falten.

Bezüglich eines einheitlichen Klassifikationssystems fand sich auch 2017 erneut keine Übereinstimmung. Dies ist am ehesten der Komplexität der Anatomie des oberen Atemwegs und den zahlreich in der Literatur beschriebenen Klassifikationssystemen geschuldet. Man einigte sich, dass ein Klassifikationssystem die Eigenschaften Obstruktionsort/anatomische Struktur, Obstruktionsgrad und Obstruktionsmuster beinhalten sollte. Ein potenzielles standardisiertes Protokoll zur Dokumentation und Beschreibung der DISE unter Einbeziehung der VOTE-Klassifikation als Grundlage mit der Möglichkeit zur Angabe weiterer relevanter Informationen, z. B. anatomischer Besonderheiten, zeigt untenstehende Box [12].

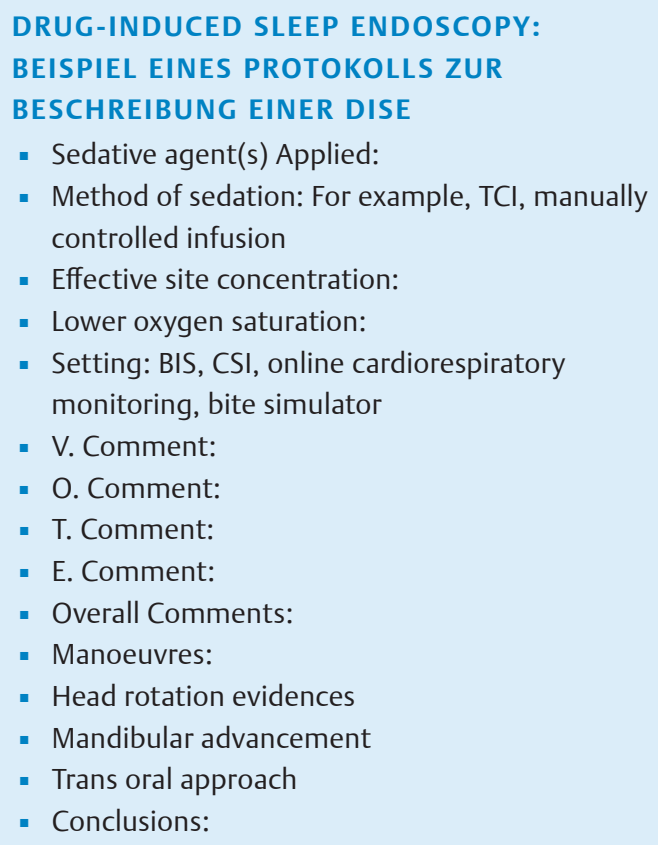

\section{Merke}

Die Kollapsorte sind der Weichgaumen, die Tonsillen und Pharynxseitenwände, der Zungengrund und die Epiglottis. Die Kollapsmuster sind antero-posterior, lateral und konzentrisch. 
- Tab. 3 und $>$ Tab. 4 zeigen weitverbreitete Klassifikationssysteme, die VOTE-Klassifikation von Kezirian [14]und die NOHL- Klassifikation von Vicini [15].

Zusammenfassend stellt die DISE eine geeignete Untersuchungsmethode zur Beurteilung des oberen Atemweges im Schlaf (medikamentös induzierter Sedierung) dar und kann somit in indizierten Fällen zur adäquaten Therapieempfehlung bei OSA beitragen [12].

\section{FALLBEISPIEL}

In der klinischen Untersuchung zeigte sich eine Tonsillenhyperplasie beidseits, ein Webbing der hinteren Gaumenbögen beidseits und eine moderate Zungengrundhyperplasie. Nach ausführlicher Beratung und Aufklärung erfolgte die medikamenten- induzierte Schlafendoskopie. Dabei konnte eine mittel- bis hochgradige Obstruktion auf Oropharynxebene nachgewiesen werden. Die hyperplastischen Tonsillen zeigten eine ausgeprägte Medialbewegung mit Verlegung des Oropharynx. Auf Velopharynxebene und auf Höhe des Zungengrundes konnte eine relevante Obstruktion ausgeschlossen werden ( $\vee$ Video 1).

\section{VIDEO 1}

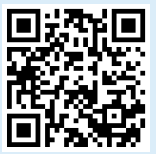

Durchführung einer Schlafendoskopie, mit Nachweis von hyperplastischen Tonsillen und einer hochgradigen Obstruktion auf Oropharynxebene.

In dem o. g. Fall konnte durch die Schlafendoskopie die bereits bei der klinischen Untersuchung angenommene Ursache der Obstruktion - die Tonsillenhyperplasie - bestätigt werden. Auch etwaige weitere Ursachen (z. B. zusätzliche Obstruktion auf Zungengrundniveau) wurden ausgeschlossen. Bei einer eindeutigen Tonsillenhyperplasie, ohne weitere anatomische Auffälligkeiten, führt die Schlafendoskopie nahezu nie zu einer Änderung der Therapie bezüglich der Tonsillen und ist somit für die Operationsindikation nicht zwingend notwendig. In Bezug auf operative Empfehlungen am Weichgaumen, Zungengrund, der Epiglottis und unter Anwendung einer Unterkieferprotrusionsschiene kann die Schlafendoskopie wichtige zusätzliche Informationen aufzeigen und gegebenenfalls zur Änderung der initialen Therapieempfehlung führen [16].

\section{Operative Therapien bei Schnarchen und obstruktiver Schlafapnoe}

Der operative Erfolg einer Therapie bei OSA wird entsprechend den Vorschlägen von Sher et al. als Reduktion des
\ Tab. 3 VOTE- Einteilung nach Kezirian 2011.

\begin{tabular}{|l|l|l|l|l|}
\hline Struktur & Grad & \multicolumn{2}{|l|}{ Konfiguration } \\
\hline & & AP & Lateral & Konzentrisch \\
\hline Velum & $0-2$ & & & \\
\hline Oropharynx & $0-2$ & - & & - \\
\hline Tonguebase (Zungengrund) & $0-2$ & & - & - \\
\hline Epiglottis & $0-2$ & & \\
\hline
\end{tabular}

Grad der Obstruktion; 0: kein Verschluss; 1: partieller Verschluss, inklusive Vibration; 2: vollständiger Verschluss; Konfiguration: Beschreibung des Verschlussmusters. AP steht für antero-posterior. Die - Felder entsprechen einem Muster, welches für die entsprechende Lokalisation nicht möglich ist.

Tab.4 NOHL-Score nach Vicini, 2012, AP - antero-posterior.

\begin{tabular}{|c|c|c|c|c|}
\hline \multirow[t]{2}{*}{ Struktur } & \multirow{2}{*}{$\begin{array}{l}\text { Grad } \\
0-25 \%: 1 \\
25-50 \%: 2 \\
50-75 \%: 3 \\
75-100 \%: 4\end{array}$} & \multicolumn{3}{|c|}{ Konfiguration } \\
\hline & & $\mathrm{AP}$ & Lateral/transversal & konzentrisch \\
\hline \multicolumn{5}{|l|}{ Nase } \\
\hline \multicolumn{5}{|l|}{ Oropharynx } \\
\hline \multicolumn{5}{|l|}{ Hypopharynx } \\
\hline Larynx & & & & \\
\hline
\end{tabular}

AHI um mindestens die Hälfte und unter einen Wert von 20 definiert [10]. Dies steht im harten Kontrast zur nächtlichen Beatmungstherapie mit einem definierten ZielRDI (Respiratory Disturbance Index- Anzahl der Apnoen und Hypopnoen sowie respiratory effort related arousals (RERA) pro Stunde Schlaf)<5/h, wobei Therapieakzeptanz, -adhärenz und -compliance ergänzend berücksichtigt werden müssen.

In der aktuellen S3-Leitlinie „Nicht erholsamer Schlaf/ Schlafstörungen“, Kapitel „Schlafbezogene Atemstörungen bei Erwachsenen“ und in der S2e-Leitlinie „HNO-spezifische Therapie der obstruktiven Schlafapnoe bei Erwachsenen “ werden zwischen resektiven und nicht-resektiven Operationsmethoden und gesichtsskelettverlagernden Verfahren (mit Osteotomien) unterschieden [1][17]. Zusätzlich unterscheidet man zwischen invasiven und minimalinvasiven Operationsverfahren. Als minimalinvasiv wird ein Eingriff angesehen, der in Lokalanästhesie und ambulant möglich ist, eine geringe peri- und postoperative Morbidität sowie geringe Komplikationsrate aufweist. Es wird auch nach primärer, sekundärer und adjuvanter Indikation unterschieden. Eine operative Maßnahme als Therapie der ersten Wahl wird zumindest als gleichwertig im Vergleich zu den apparativen Verfahren angesehen. Nach 


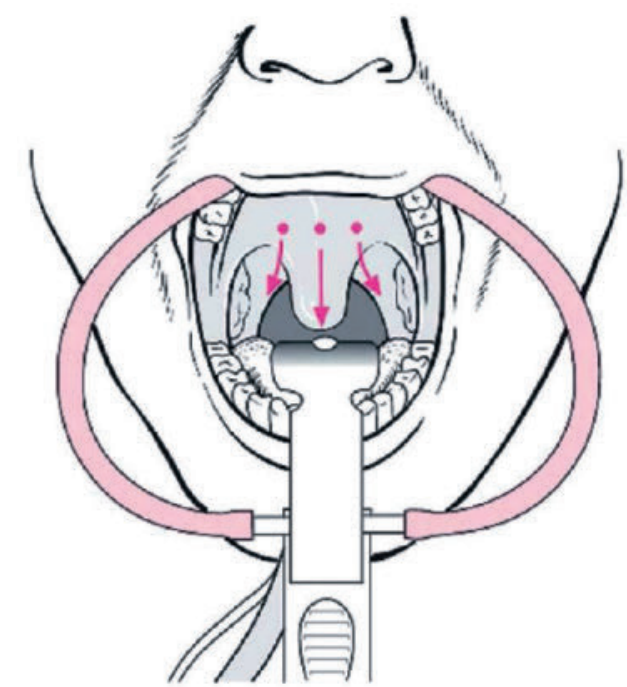

- Abb. 2 Interstitielle Radiofrequenztherapie (RFT) des Weichgaumens. Eingezeichnet sind die Inzisionspunkte und Stichrichtung in Uvula und Weichgaumen. Aus: Theissing J, Rettinger G, Werner JA. HNO-Operationslehre. 4. Aufl. Stuttgart: Thieme; 2006.

erfolgloser apparativer Therapie kann eine operative Therapie der zweiten Wahl erwogen werden. Eine adjuvante Operation unterstützt den Erfolg der primären Therapie ohne - in Bezug auf die OSA - für sich alleine erfolgreich zu sein [17].

\section{Merke}

Operative Therapien bei gutem Allgemeinzustand des Patienten kommen infrage, wenn ein korrigierbarer pathologischer Befund besteht und präoperativ sorgfältig diagnostiziert wurde.

\section{Verbesserung der Nasenatmung}

Eine alleinige Nasenoperation (Septumplastik, Nasenmuschelplastik, Septorhinoplastik und Nasennebenhöhlenoperation) kann den $\mathrm{AHI}$ nicht signifikant senken und kann zur primären Behandlung der OSA nicht empfohlen werden. Eine Nasenoperation kann jedoch eine relevante Nasenatmungsbehinderung beseitigen und die Schlafqualität und Erholungsfunktion des Schlafes primär verbessern. Zusätzlich kann sie als adjuvante Therapie die CPAP-Compliance verbessern und den Therapiedruck reduzieren [17].

\section{Minimalinvasive Verfahren beim Schnarchen und bei geringgradiger OSA}

Minimalinvasive Interventionen sind die interstitielle Radiofrequenztherapie (RFT) mit hochfrequentem Strom und der Einsatz von Weichgaumenimplantaten. Es kön- nen mehrere minimalinvasive Methoden kombiniert werden.

\section{Interstitielle Radiofrequenztherapie (RFT)}

Die RFT wird am Weichgaumen, den Tonsillen und am Zungengrund eingesetzt ( $>$ Abb. 2). Ziel ist die narbige Versteifung der Muskulatur von Weichgaumen und Zunge durch Teilnekrose im Gewebe mit folgender Fibrosierung. Im lymphatischen Gewebe der Tonsillen findet sich zusätzlich ein Volumeneffekt von bis zu 75\% [18]. Der geringe postoperative Wundschmerz und die geringen Komplikationen sind durch die Schonung der Mukosa bedingt. Die wichtigsten Komplikationen sind:

- Ulzera

- Einblutung

- Odynophagie [19].

\section{Weichgaumenimplantate}

Weichgaumenimplantate beabsichtigen den Weichgaumen durch die induzierte Vernarbung zu versteifen und somit Vibrationsbewegungen zu reduzieren. > Video 2 zeigt Vibrationen des Weichgaumens. In den Weichgaumen können nichtresorbierbare zylindrische Kunststoffimplantate (Polyethylenterephthalat) in Lokalanästhesie appliziert werden. Insgesamt werden 3 Implantate in den Weichgaumen eingebracht. Gemäß S3-Leitlinie schlafbezogener Atmungsstörungen sind für Weichgaumenimplantate eine geringe bis moderate Wirksamkeit für die Reduktion des Schnarchens und der OSA nachgewiesen [1][17].

\section{VIDEO 2}

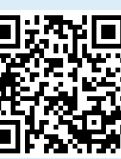

Schlafendoskopie mit Nachweis von Vibrationen des Weichgaumens.

\section{Merke}

Eine primäre Indikation für minimalinvasive Verfahren ergibt sich bisher nur beim einfachen Schnarchen.

Beim OSA ist der primäre Einsatz an Weichgaumen und Zungengrund allenfalls für die milde OSA indiziert.

\section{Invasive Therapieverfahren bei OSA}

\section{Weichgaumen und Oropharynx \\ Uvulopalatoplastik (UPP)}

Die Uvulopalatoplastik (UPP) stellt ein schleimhaut-resezierendes Verfahren ohne plastische Nähte dar. Sie kann in der Regel ambulant in Lokalanästhesie durchgeführt werden. Die UPP kann mit dem Laser, der Elektrochirurgie, dem Mikrodebrider oder anderen technischen Hilfsmitteln ausgeführt werden ( $\triangleright$ Abb. $\mathbf{3}$ ). In mehreren systematischen Reviews wurde die UPP in Bezug auf Reduktion des 
AHI, Nebenwirkungen und Komplikationsrate als nicht indiziert für die Behandlung der OSA angesehen. Das Verfahren wird daher in der S2e-Leitlinie „HNO-spezifische Therapie der obstruktiven Schlafapnoe bei Erwachsenen “ für die Behandlung der OSA nicht empfohlen [17]. Die S2kLeitlinie „Diagnostik und Therapie des Schnarchens des Erwachsenen“ empfiehlt jedoch die UPP zur Behandlung des Schnarchens [5].

\section{Uvulopalatopharyngoplastik (UPPP)}

Die etablierteste chirurgische Intervention ist die Uvulopalatopharyngoplastik, kurz UPPP. Das Prinzip besteht in der Erweiterung des oropharyngealen Raumes in transversaler als auch in sagittaler Richtung. Sie wurde erstmals 1964 von Ikematsu zur Behandlung des Schnarchens beschrieben [21]. Die UPPP zeichnet sich durch eine sehr muskelschonende Operationstechnik aus, mit dem Ziel der Reduktion des Schnarchens. 1981 wurde von Sullivan et al. die CPAP- Therapie und von Fujita et al. die modifizierte UPPP zur Behandlung der OSA vorgeschlagen. Die ersten OP-Techniken zur Behandlung der OSA waren radikaler als die ursprüngliche Technik von Ikematsu. Es wurden die freie Uvula und Anteile der Gaumenbogenmuskulatur reseziert. Folgenschwere Komplikationen waren die Nasopharynxstenose und die velopharyngeale Insuffizienz. Als Erste in Deutschland erkannten Pirsig et al., dass eine radikale chirurgische Therapie nur mit erhöhter Komplikationsrate einhergeht, jedoch nicht die Erfolgsrate der UPPP bei OSA verbessert. Aus diesen Erkenntnissen leitete Pirsig seine Operationstechnik ab. Die Operationstechnik basierte auf:

- der Schonung aller velaren Muskeln

- der Formung eines weniger vibrierenden Velums

- der Vorverlagerung der Velumkante

- der Lateralisation der hinteren Gaumenbögen

- der pharyngealen Volumenerweiterung durch eine Tonsillektomie (TE) ( $\mathbf{A b b . 4 ) . ~}$

Zusätzlich wurde die Wundheilung durch den Einsatz resorbierbarer Fäden, zum Beispiel 3-0 Vicryl, verbessert [22].

Die TE-UPPP ist im Vergleich zu anderen Weichteiloperationen im HNO-Gebiet die Operation mit dem höchsten abgesicherten Evidenzgrad. Sommer et al. konnten in einer randomisierten kontrollierten klinischen Studie zeigen, dass der AHI bei über $90 \%$ der operierten Patienten gesenkt werden konnte. Insgesamt konnte eine $54 \%$ ige Reduktion des $\mathrm{AHI}$ in der Interventionsgruppe im Vergleich zur Kontrollgruppe nachgewiesen werden. Nach den SherKriterien konnte bei über $70 \%$ der Interventionsgruppe ein chirurgischer Erfolg nachgewiesen werden [23]. Dies deckt sich auch mit den Daten von Browaldh et al., welche eine Reduktion des AHI nach TE-UPPP von $60 \%$ nachweisen konnten. Beide Studien tragen zum Nachweis der Effektivität der TE-UPPP zur Behandlung der OSA bei. Insbesondere im Vergleich zu einer Metaanalyse von 2010,

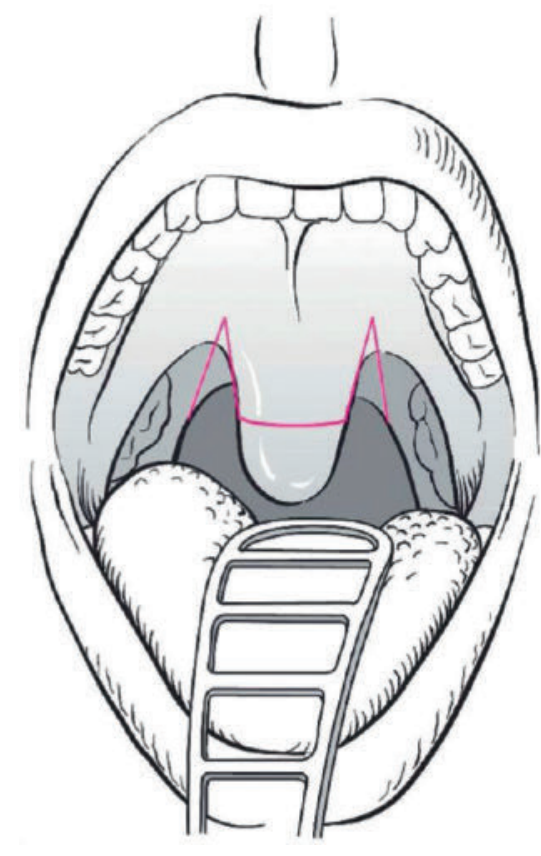

- Abb. 3 Laser-assistierte Uvulopalatoplastik (LAUP). Schema zur Lokalisation der Inzisionen mit dem Laser parauvulär und an der Uvula zur Uvulakürzung. Aus: Theissing J, Rettinger G, Werner JA. HNO-Operationslehre. 4. Aufl. Stuttgart: Thieme; 2006.

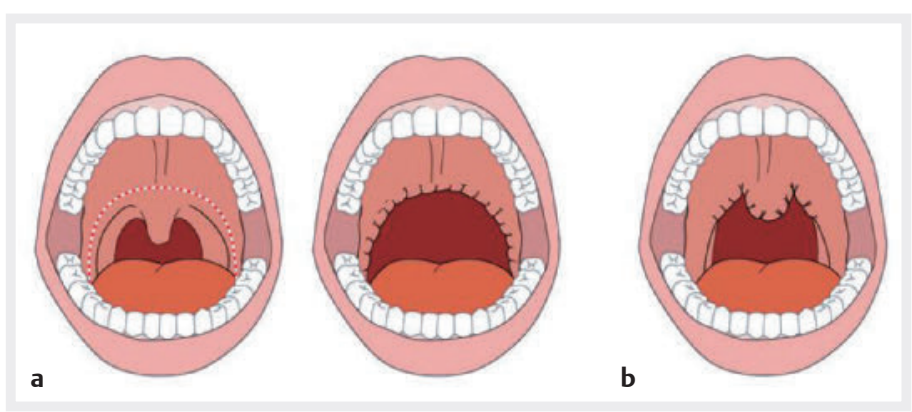

- Abb.4 Uvulopharyngopalatoplastik (UPPP). Prinzip der Gaumenbogenraffung und Uvulakürzung. a radikale Technik, b funktionelle Technik. Aus: Zahnert T. Schlafbezogene Atmungsstörungen. Laryngo-RhinoOtologie 2011; 90 (11): 691-707.

in der 15 Kohortenstudien mit durchgeführter UPPP verglichen wurden und eine Reduktion des AHI um 33\% nachgewiesen wurde [23][24]. Zusätzlich konnte in den Arbeiten von Sommer et al. und Browaldh et al. aufgezeigt werden, dass sich auch die Tagesschläfrigkeit, Schnarchen und weitere respiratorische Parameter signifikant besserten. In der kürzlich publizierten systematischen Übersichtsarbeit von Stuck et al. variierten die Erfolgsraten der TE-UPPP zwischen 31,3 und 96\%. Zusätzlich konnte auch eine Reduktion der Schläfrigkeit anhand des prä- und postoperativ erfassten Mittelwerts der Epworth Sleepiness Scale (ESS) aufgezeigt werden. Es konnte insgesamt gezeigt werden, dass die UPPP bezüglich einer Reduktion des AHI als auch 
des ESS gegenüber einer Kontroll- oder unbehandelten Gruppe signifikant überlegen ist. Bezüglich des Langzeiteffekts und des Einflusses auf kardiovaskuläre Mortalität sollten weitere Studien zur differenzierten Erfassung erfolgen [25].

Der Effekt der UPPP auf den Schweregrad der OSA kann mit den Jahren nachlassen. Der Langzeiterfolg sinkt von 60,5\% nach 3-12 Monaten auf 47,6\% nach 3-7 Jahren [17]. Patienten nach UPPP sollten längerfristig schlafmedizinisch kontrolliert werden [17].

Merke

Die UPPP mit TE hat den höchsten abgesicherten Evidenzgrad der chirurgischen Therapie bei OSA und wird bei entsprechendem anatomischem Befund zur Therapie der OSA empfohlen.

Vor dem Hintergrund der Verbesserung der Langzeitergebnisse werden seit Jahren verschiedene Modifikationen der UPPP entwickelt, ohne dass sich eine neue Standardtechnik erkennen lässt. Die Grundprinzipien der Modifikationen unterscheiden zwischen Lappentechniken, Erweiterungsplastiken der lateralen Pharynxwand, Z-Plastiken und anderen Techniken [17]. Für weiterführende Informationen wird auf die aktuelle Literatur verwiesen.

\section{Zungengrund und Hypopharynx}

Im Gegensatz zur Chirurgie am Weichgaumen und Oropharynx gibt es keine etablierte Standardtechnik zur operativen Behandlung der retrolingualen und hypopharyngealen Obstruktion. Die meisten Verfahren werden in Kombination mit anderen Eingriffen eingesetzt. Diese Methode nennt man auch Multi-Level-Chirurgie. Im Folgenden werden die Eingriffe erklärt. Für weiterführende Informationen wird auf die Literatur verwiesen.

\section{Hyoidsuspension}

Ziel der Hyoidsuspension ist die Vorverlagerung des Zungenbeines mit der daran befestigten Muskulatur. Hierfür wird das Zungenbein entweder mit dem Kinn (Hyoidsuspension Typ 1) oder mit dem Schildknorpel (Hyoidsuspension Typ 2) verbunden. In einer Fall-Kontroll-Studie von 2014 konnte eine Reduktion des AHI von 43/h auf 11/h nachgewiesen werden. Die chirurgische Erfolgsrate nach Sher wurde somit mit $76 \%$ angegeben [17].

\section{Zungensuspension}

Die Zungensuspension verfolgt das gleiche Ziel wie die Hyoidsuspension, das Verhindern des nach-hinten-Fallens der Zunge im Schlaf. Initial wurde als Zügel autologe Faszia lata benutzt. Diese Methode konnte sich aber aufgrund der aufwendigen Präparation nicht durchsetzen. Durch Einführung des Repose ${ }^{T M}$ Systems (Medtronic, USA) wurde die Methode wieder aufgegriffen. Mithilfe eines Applikator-Systems wird der Zungengrund durch nicht-resorbierbares monophiles Nahtmaterial an eine auf der Kinnin- nenseite eingebohrte Titanschraube gezügelt. Belastbare Studienergebnisse liegen für dieses Verfahren aktuell noch nicht vor [17].

\section{Larynx und Trachea \\ Epiglottis}

Die isolierte laryngeale OSA ist im Erwachsenenalter selten. Malformationen der Epiglottis führen zu einer Instabilität, infolge derer die Epiglottis bei Inspiration auf den Larynx bzw. gegen die Rachenhinterwand gesogen wird. Die Diagnose wird mittels Schlafendoskopie gestellt und kann Grund für eine CPAP (continuous positive airway pressure)-Masken-Intoleranz sein ( $\vee$ Video 3). Die „Floppy Epiglottis “ wird eher beim älteren Patienten beobachtet, kann aber auch jüngere Erwachsene betreffen. Die Therapien der Wahl sind die (Laser-)chirurgische Teilresektion der Epiglottis, Laserstichelung der lingualen Epiglottisfläche bzw. Straffung des Ligamentes der Plica glossoepiglottica mediana. Alternativ - nicht chirurgisch - kann die Anwendung einer Unterkieferprotrusionsschiene (UKPS) erwogen werden [17].

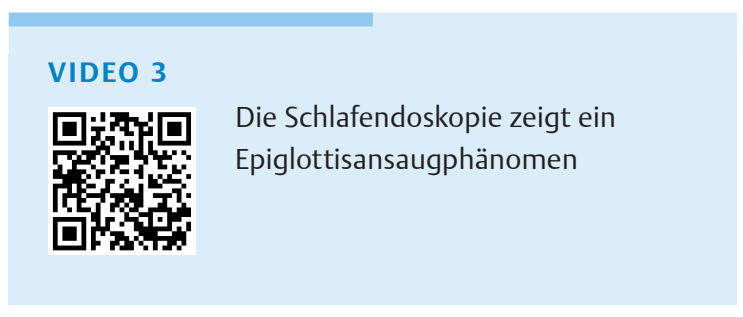

\section{Tracheotomie}

Die Tracheotomie ist die älteste und erfolgreichste Behandlungsoption der OSA, mit einer Erfolgsquote von annähernd $100 \%$. Sie wird aufgrund der Einschränkung der Lebensqualität als ultima ratio in ausgewählten Fällen eingesetzt. Die Indikation ist streng zu stellen [17].

\section{Nervenstimulation}

\section{Stimulation des Nervus Hypoglossus während des Schlafes}

Alle Systeme zur Hypoglossusnervstimulation müssen chirurgisch implantiert werden. Sie sind bisher ausschließlich für Patienten gedacht, die eine CPAP-Therapie nicht vertragen bzw. damit nicht erfolgreich zu behandeln sind (sogenannte CPAP-Versager). Die Therapie basiert auf der zum Teil selektiven Stimulation des N. hypoglossus, welcher den wesentlichen Atemwegsöffner, den M. genioglossus innerviert. Die insuffiziente Tonisierung der pharyngealen Muskulatur soll somit während des Schlafes wiederhergestellt werden. 1996 wurde das Konzept erstmalig am Menschen angewandt. Es wurden 2 Systeme zur Markreife entwickelt:

- Inspire ${ }^{\mathrm{TM}}$ Upper Airway Stimulation System (Fa. Inspire Medical System, Maple Grove/MN, USA) 


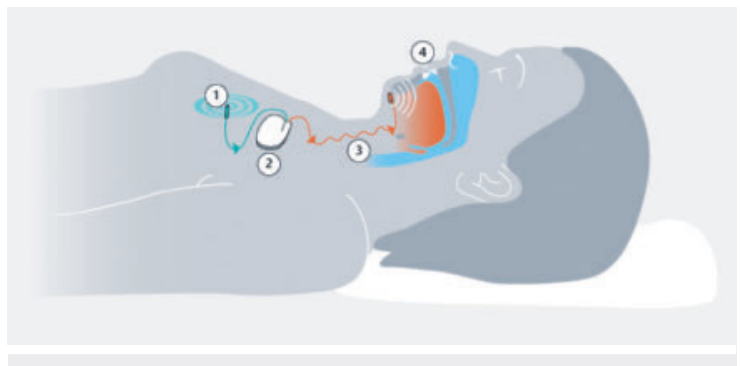

Abb. 5 Schematische Darstellung der Nervus-Hypoglossus-Stimulation. Der Sensor (1) erkennt die einzelnen Atemzüge des Patienten. Der Pulsgenerator (2) verarbeitet die Atmungsdaten und generiert die Stimulation. Die Stimulation des oberen Atemwegs (3) verhindert, dass der Atemweg während der Atmung kollabiert. Der Atemweg wird somit während der Therapie offengehalten (4).

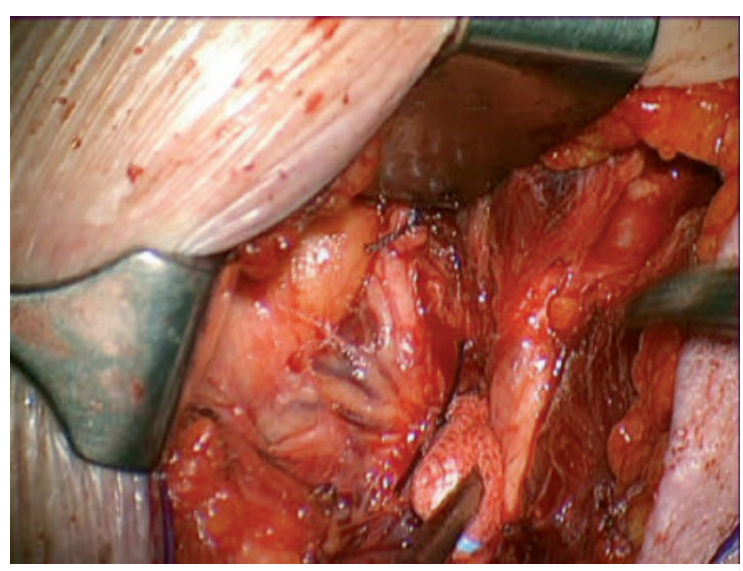

- Abb. 6 Darstellung der Aufgliederung des N. hypoglossus in den medialen und lateralen Anteil. Um eine adäquate Protrusion der Zunge auszulösen, ist diese Präparation gefordert. Aus: Steffen A, Heiser $C$ et al. Stellungnahme der Taskforce „Neurostimulation bei Schlafapnoe“ zur Stimulation der oberen Atemwege. Laryngo- RhinoOtologie 2015; 94(04): 221-224.

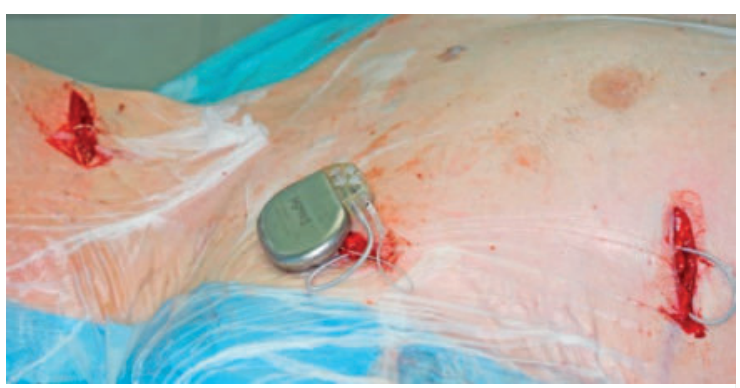

- Abb. 7 Intraoperative Situation am Beispiel der atmungsgesteuerten Stimulation: Die Stimulationselektrode vom submentalen Zugang und die Sensorsonde aus dem Interkostalbereich sind mit der klavikulären Impulsgeneratortasche verbunden. Vor Wundverschluss erfolgt der telemetrische Check mit einer ersten Teststimulation. Aus: Steffen A, Heiser C et al. Stellungnahme der Taskforce "Neurostimulation bei Schlafapnoe“ zur Stimulation der oberen Atemwege. Laryngo- Rhino- Otologie 2015; 94(04): 221-224.
- ImThera aura6000'M Targeted Hypoglossus Stimulation System (Fa. LivaNova, London, UK))

Beiden Systemen gemeinsam ist die subkutane Implantation einer Nervenstimulationseinheit und die direkte Stimulation des N. hypoglossus durch eine anliegende Stimulationselektrode.

Das ImThera-THN-System stimuliert die oberen Atemwege atmungsunabhängig und kontinuierlich. Dadurch soll eine Versteifung des oberen Atemwegs erreicht werden, sodass dieser nicht kollabiert. Die Stimulationselektrode ist am Hauptstamm des N. hypoglossus lokalisiert. In einer 2017 publizierten Studie konnte eine Reduktion des AHI von 34,9/h $\pm 22,5 / \mathrm{h}$ auf $25,4 / \mathrm{h} \pm 23,1 / \mathrm{h}$ beobachtet werden. In der Gruppe mit den Schlüsselprädiktoren $\left(\mathrm{BMI}<35 \mathrm{~kg} / \mathrm{m}^{2}, \mathrm{AHI}<65 / \mathrm{h}\right.$, Apnoe-Index $(\mathrm{Al})<30 / \mathrm{h}$ und das Auftreten eines mehr als 10 \%igen Abfalls der Sauerstoffsättigung von weniger als 15/h) konnte der AHI sogar von $35,7 / h \pm 19,4 / h$ auf $8,5 / h \pm 5,9 / h$ gesenkt werden. Langzeitergebnisse und größere multizentrische Studien sind bislang nicht verfügbar [17][27].

Das atmungsgesteuerte Inspire-System benötigt zur wirksamen Stimulation einen Sensor, welcher den Atemrhythmus des Patienten registriert. Die Stimulation des Hypoglossusnerves wird in der späten Exspiration ausgelöst und hält die gesamte Inspiration an ( $\triangleright$ Abb. 5) ( $\triangleright$ Abb. 7). Es werden die distalen Fasern des N. hypoglossus stimuliert ( Abb.6). Dadurch wird eine Protrusion der Zunge, insbesondere der Zungenbasis, ausgelöst, sodass sich der Atemweg auf Höhe des Zungengrundes öffnet. Zusätzlich kann eine Öffnung auf Weichgaumenebene beobachtet werden. Vermutet wird eine muskuläre Kopplung von Zungenbasis und Velopharynx über den M. palatoglossus. Das InspireSystem wird empfohlen, wenn ein vollständiger konzentrischer Kollaps retropalatal ausgeschlossen wurde. Zusätzlich sollte der AHI zwischen 15/h und 65/h liegen, der $\mathrm{BMI} \leq 35 \mathrm{~kg} / \mathrm{m}^{2}$ und die zentral/gemischten Apnoen $\leq 25 \%$ sein. Nach Implantation ist die schlafmedizinische Kontrolle mittels PG oder PSG 3-6 Monate postoperativ erforderlich. Studien zeigten während eines Beobachtungszeitraumes von 12 Monaten eine Reduktion des medianen $\mathrm{AHI}$ um $68 \%$. Ebenso war die Therapieadhärenz mit 86 \% sehr hoch. 2015 bzw. 2017 wurden Langzeitergebnisse publiziert, in denen sich die Reduktion des AHI auch nach 30 sowie 60 Monaten beständig zeigte. In der bis jetzt größten internationalen Datenauswertung konnte eine Reduktion des AHI von 36,3 $\pm 15,7 / \mathrm{h}$ auf $10,3 \pm 11,5 / \mathrm{h}$ nachgewiesen werden [27][28].

\footnotetext{
Merke

Die atmungssynchrone Stimulation des N. hypoglossus kann bei mittel bis schwerer OSA und Ineffektivität/ Unverträglichkeit der CPAP-Therapie empfohlen werden. Eine suffiziente Diagnostik mittels Schlafendoskopie ist obligat.
} 


\section{Gesichtsskelettverlagernde Verfahren}

Nicht primär den HNO-Facharzt betreffend gibt es weitere operative Möglichkeiten zur Behandlung der OSA.

\section{Maxillomandibuläre Osteotomie (MMO)}

Die Durchgängigkeit des oberen Atemwegs wird maßgeblich durch das Gesichtsskelett, die Kieferform und Position bestimmt. Die MMO erweitert gleichzeitig Naso-, Oro- und Hypopharynx durch Vorverlagerung von Weichgaumenstrukturen, Zunge und Tonuserhöhung der pharyngealen Muskulatur. Die MMO gilt heute als Standardeingriff, bleibt jedoch technisch anspruchsvoll und zieht weitere stationäre Nachbehandlungen nach sich [17][29].

\section{Merke}

Die MMO ist nach der Tracheotomie das erfolgreichste operative Verfahren und wird der CPAP-Therapie mit vergleichbarer Reduktion des AHI und Optimierung der Schlafarchitektur gleichwertig gesetzt.

Die MMO ist bei Patienten mit OSA und entsprechender Anatomie indiziert. Bei Indikationsstellung müssen Komplikationen, Morbidität und vor allem kosmetische Konsequenzen beachtet werden [29].

\section{Zusammenfassung}

Schlafbezogene Atmungsstörungen sind heutzutage von großer klinischer und sozialer Bedeutung. Die Diagnostik und Therapie der OSA und somit die Reduktion der potenziellen kardiovaskulären Risiken, aber auch der Vigilanzstörungen und der Folgen stehen im Fokus vieler beteiligter Fachdisziplinen. Während im Kindesalter die Domäne der Therapie der OSA in der chirurgischen Behandlung liegt, sollte der Therapieansatz im Erwachsenenalter nach der Ursache, Lokalisation und Prognose der Obstruktion individuell und differenzierter betrachtet werden. Über die primäre Intervention bei OSA wird kontrovers diskutiert. Die hohe Erfolgsquote bei besserer Studienlage sprechen für die Beatmungstherapie und gegen die chirurgische Therapie. Allerdings ist die CPAP-Akzeptanz, insbesondere bei gering- und mittelgradiger OSA ohne relevante Symptome besonders gering, weshalb in dieser Patientengruppe die operative Therapieoption so beliebt ist. Insbesondere sind ein niedriger Ausgangswert im AHI und BMI sowie geringe Tagessymptomatik positive Prädiktoren für einen Operationserfolg. Je geringer die OSA, desto weniger umfangreich und invasiv gestaltet sich die operative Therapie und somit die peri- und postoperative Morbidität und Komplikationsrate [3].

Zusammenfassend stellt nach aktuellen Kenntnissen weiterhin die Beatmungstherapie den Goldstandard zur Therapie der mittleren bis schweren OSA dar. Jedoch bieten chirurgische Therapieoptionen nach sorgfältiger Diagnostik im selektionierten Patientengut zuverlässige Alternati- ven zur CPAP-Therapie bzw. bei CPAP-Intoleranz suffiziente Behandlungsstrategien.

\section{KERNAUSSAGEN}

- Die Prävalenz der milden OSA beträgt $20 \%$, der mittel- bis hochgradigen OSA ca. 6-7\% beim Erwachsenen.

- Goldstandard zur Therapie der mittel- bis hochgradigen OSA ist die Ventilationstherapie mit CPAP-Maske. Diese ist bei guter Compliance und Therapieadhärenz erfolgreich.

- Die chirurgische Therapie bietet im Gegensatz zur CPAP-Therapie eine $100 \%$ ige Therapieadhärenz.

- Zur Behandlung des Schnarchens und der gering- bis mittelgradigen OSA stehen verschiedene Operationsverfahren zur Verfügung.

- Die chirurgische Therapie setzt eine adäquate präoperative Diagnostik voraus. Diese beinhaltet in jedem Fall eine schlafmedizinische und klinisch-endoskopische Untersuchung, welche durch die Schlafendoskopie ergänzt werden kann.

- Der operative Erfolg einer Therapie bei OSA wird entsprechend Sher et al. als Reduktion des AHI um mindestens die Hälfte und unter einen Wert von 20 definiert. Diese steht im Kontrast zur nächtlichen Beatmungstherapie mit einem definierten Ziel-Wert<5/h, wobei Therapieakzeptanz, -adhärenz und -compliance ergänzend berücksichtigt werden müssen.

- Bei schwerer OSA sollten operative Maßnahmen nur zur Optimierung der Beatmungstherapie bzw. bei Intoleranz oder mangelnder Adhärenz durchgeführt werden.

Falls nun Ihr Interesse geweckt ist, ist jeder zur aktiven Mitarbeit in der AG Schlafmedizin der Deutschen Gesellschaft für Hals-, Nasen- und Ohrenheilkunde, Kopf-HalsChirurgie eingeladen.

\section{Interessenkonflikt}

\section{Erklärung zu finanziellen Interessen}

Die Autorinnen/Autoren geben an, dass kein Interessenkonflikt besteht.

Erklärung zu nichtfinanziellen Interessen

Die Autorinnen/Autoren geben an, dass kein Interessenkonflikt besteht. 


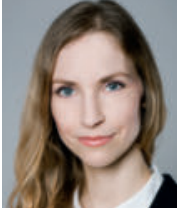

\section{Maren Just}

Dr. med., 2005-2011 Medizinstudium Universität Leipzig, 2016 Promotion, Fachärztin für HNO-Heilkunde (2017), Zusatzqualifikation Allergologie (2018). Seit 2017 Leiterin der Poliklinik, Klinik der Hals-, Nasen-, Ohrenheilkunde Universitätsklinikum Leipzig. Betreuung der allergologischen Sprechstunde und schlafmedizinischen Sprechstunde/Operationen.

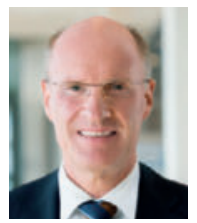

\section{Andreas Dietz}

Seit April 2004 ist Prof. Dr. Andreas Dietz Direktor der HNO-Klinik des Universitätsklinikums Leipzig. Während seiner Zeit als Assistenz- und Oberarzt arbeitete er an der HNO-Universitätsklinik für HNO in Heidelberg. Er ist ausgebildeter HNO- und Kopf-HalsChirurg und verfügt über spezielle Weiterbildungen plastische Operationen, spezielle Kopf-Hals-Chirurgie, Medikamentöse Tumortherapie und Allergologie. Im Rahmen zahlreicher Studien und translationalen Projekten konzentrierte sich seine wissenschaftliche Arbeit auf Kopf-Hals-Tumoren und ist international als Experte auf diesem Gebiet bekannt. Seit 2013 ist er Mitglied des Präsidiums und aktuell Präsident der Deutschen Gesellschaft für Hals-, Nasen- und Ohrenheilkunde. Er ist in zahlreichen internationalen Gremien in sichtbaren Positionen tätig. Er ist n. a. Mitglied des Board of Directors der European Head and Neck Society. Er ist Ehrenmitglied der Königlich Belgischen HNO-Gesellschaft, der Österreichischen und tschechischen HNO-Gesellschaft und der Indian Head and Neck Foundation. Er ist Vorstandsmitglied des Innovationszentrums für Computerassistierte Chirurgie (ICCAS) Leipzig. Er ist Mitglied des Board of Directors des „Global Online Fellowship Initiative“ des Sloan Kettering Memorial Cancer Centers in New York und Vorsitzender des Unterausschusses Chirurgie der EORTC Head and Neck Group. Andreas Dietz ist Autor/ Co-Autor von mehr als 360 pub-med gelistete Publikationen.

\section{Korrespomndenzadresse}

Dr. med. Maren Just

Universitätsklinikum Leipzig, HNO-Klinik

Liebigstr. 10 - 14

04103 Leipzig

maren.just@medizin.uni-leipzig.de

\section{Wissenschaftlich verantwortlich gemäß Zertifizierungsbestimmungen}

Wissenschaftlich verantwortlich gemäß Zertifizierungsbestimmungen für diesen Beitrag ist Dr. med. Maren Just
[1] Mayer G, Arzt M, Braumann B et al. S3- Leitlinie Nicht erholsamer Schlaf/Schlafstörungen, Kapitel "Schlafbezogene Atmungsstörungen bei Erwachsenen". Im Internet: www. awmf.org/uploads/tx_szleitlinien/063-001I_S3_SBAS_201708_2.pdf

[2] Wilhelm I, Rose M, Imhof KI et al. The sleeping child outplays the adult's capacity to convert implicit into explicit knowledge. Nat Neurosci 2013; 16: 391-393

[3] Verse T, Hörmann K. Operative Therapie der Obstruktion bei schlafbezogenen Atemstörungen. Dt. Ärzteblatt 2011; 108; 13: $216-221$

[4] Zahnert Th. Schlafbezogene Atmungsstörungen. LaryngoRhino- Otologie 2011; 90: 691-707

[5] Stuck BA et al. S2k-Leitlinie „Diagnostik und Therapie des Schnarchens des Erwachsenen “. Im Internet: https://www. awmf.org/uploads/tx_szleitlinien/017-068I_S2k_Leitlinie Schnarchen_2013-verlaengert_01.pdf

[6] Pieters T, Collard P, Aubert G et al. Acceptance and longterm compliance with nCPAP in patients with obstructive sleep apnoea syndrome. Eur Respir J 1996; 9:939-9446.

[7] Grote L, Hedner ], Grunstein R, Kraiczi H. Therapy with nCPAP: incomplete elimination of sleep related breathing disorder. Eur Respir ] 2000; 16:921-9277.

[8] Ravesloot MJL, de Vries N, Stuck BA. Treatment adherence should be taken into account when reporting treatment outcomes in obstructive sleep apnea. Laryngoscope 2014; 124:344-3458.

[9] Stuck BA, Leitzbach S, Maurer JT. Effects of continuouspositive airway pressure on apnea-hypopnea index in obstructive sleep apnea based on long-term compliance. Sleep Breath 2010; 16:467-4719.

[10] Sher AE, Schechtman KB, Piccirillo JF. The efficacy of surgical modifications of the upper airway in adults with obstructive sleep apnea syndrome. Sleep 1996; 19:156-177

[11] Borowieki B, Pollak CP, Weitzman ED et al. Fibrooptic study of pharyngeal airway during sleep in patients with hypersomnia obstructive sleep- apnoe syndrome. Laryngoscope 1978; 88: 1310-1313

[12] De Vita A, Carrasco Llatas M, Ravesloot M], et al. European position paper on drug-induced sleep endoscopy: $2017 \mathrm{Up}$ date. Clin Otolaryngol. 2018; 43:1541-1552

[13] Widmer G, Tschopp K, Kneschke TO. Alternativen zur CPAPTherapie? Die Schlafendoskopie hilft bei der richtigen Wahl. Swiss Medical Forum 2016; 16(11): 273-278

[14] Kezirian E], Hohenhorst W, de Vries N. Drug-induced sleep endoscopy: the VOTE classification. Eur Arch Otorhinolaryngol 2011; 268:1233-1236

[15] Vicini C, De Vito A, Benazzo M et al. The nose oropharynx hypopharynx and larynx (NOHL) classification: a new system of diagnostic standardized examination for OSAHS patients. Eur ArchOtorhinolaryngol 2012; 269:1297-1300

[16] Eichler C, Sommer JU, Stuck BA, Hörmann K, Maurer JT. Does drug-induced sleep endoscopy change the treatment concept of patients with snoring and obstructive sleep apnea? Sleep Breath 2013;17(1): 63-8.

[17] Verse T; Bodlaj R, de la Chaux R, et al.: ArGe Schlafmedizin der Deutschen Gesellschaft für Hals-Nasen-Ohren-Heilkunde, Kopf- und Hals-Chirurgie. Sze- Leitlinie: HNO- spezifische Therapie der obstruktiven Schlafapnoe bei Erwachsenen. HNO 09/2015 
[18] Nelson LM. Temperature-controlled radiofrequency tonsil reduction in children. Arch Otolaryngol Head Neck Surg 2003; 129: 533-7

[19] Toh ST, Hsu PP, Ng YH, Teo TW, Tan KL, Lu KS. Incidence of complications after temperature-controlled radiofrequency treatment for sleep-disordered breathing: a Singapore sleep centre experience. J Laryngol Otol 2008; 122: 490-4

[20] Maurer JT, Verse T, Stuck BA, Hörmann K, Hein G. Palatal implants for primary snoring: short-term results of a new minimally invasive surgical technique. Oto-laryngol Head Neck Surg 2005; 132: 125-31

[21] Ikematsu T. Study of snoring. Therapy. J Jpn Otol Rhinol Laryngol Soc 1964;64:434-435

[22] Pirsig W, Schäfer J, Yildiz F, Nagel J. Uvulopalatopharyngoplastik ohne Komplikationen: eine Modifikation nach Fujita. Laryngorhinootologie 1989; 68:585-590

[23] Sommer JU, Heiser C, Gahleitner C, Herr RM, Hörmann K, Maurer JT, Stuck BA: Tonsillectomy with uvulopalatopharyngoplasty in obstructive sleep apnoe- a two center randomized controlled trail. Dtsch Arztebl Int 2016; 113: 1-8.

[24] Browaldh N, Nerfeldt P, Lysdahl M, Bring J, Friberg D: SKUP3 randomised controlled trial: polysomnographic results after uvulopalatopharyngoplasty in selected patients with obstructive sleep apnoea. Thorax 2013; 68: 846-53.

[25] Stuck BA, Ravesloot M], Eschenhagen T, Sommer JU: Tonsillektomie mit Uvulopalatopharyngoplastik zur Behandlung der obstruktiven Schlafapnoe des erwachsenen. Ein systematischer Review in der Kurzfassung. Somnologie 2018; 02.

[26] Maurer JT. Update on surgical treatments for sleep apnea. Swiss Med Wkly 2009; 139:624-629

[27] Heiser C, Hofauer B. Hypoglossusnervstimulation bei CPAPVersagen. HNO 2017; 65: 99-106

[28] Heiser $C$ et al. Post-approval upper airway stimulation predictors of treatment effectiveness in the ADHERE registry. Eur Respir J 2019; 53: 1801405

[29] Conradt R, Hochban W, Heitmann J, et al. Sleep fragmentation and daytime vigilance in patients with OSA treated by surgical maxillomandibular advancement compared to CPAP therapy. J Sleep Res 1998;7: 217-23

Bibliografie

DOI https://doi.org/10.1055/a-0901-9863

Laryngo-Rhino-Otol 2019; 98: 638-650

(c) Georg Thieme Verlag KG Stuttgart · New York

ISSN 0935-8943 


\section{Punkte sammeln auf CM/F.thieme.de}

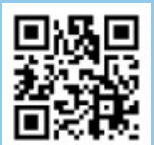

Diese Fortbildungseinheit ist in der Regel 12 Monate online für die Teilnahme verfügbar.

Den genauen Einsendeschluss finden Sie unter https://eref.thieme.de/CXD1IP7.

Sollten Sie Fragen zur Online-Teilnahme haben, finden Sie unter https://cme.thieme.de/hilfe eine ausführliche Anleitung. Wir wünschen viel Erfolg beim Beantworten der Fragen!

Unter https://eref.thieme.de/CXD1IP7 oder über den QR-Code kommen Sie direkt zur Startseite des Wissenstests.

VNR 2760512019156640227

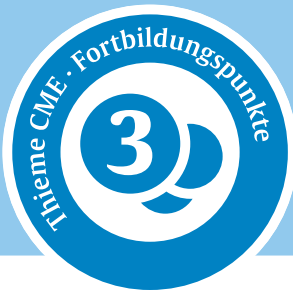

\section{Frage 1}

Welche Aussage ist falsch?

A Korrekturen der Nasenatmung können zur Erleichterung der Maskenbeatmung sinnvoll sein.

B Eine alleinige Operation zur Verbesserung der Nasenatmung kann zur Behandlung der OSA ausreichend sein.

C Die Tonsillektomie hat auch im Erwachsenenalter eine gute Erfolgsrate der Behandlung der OSA.

D Bei der Chirurgie des Weichgaumens haben sich heute funktionserhaltende gegenüber radikalen Operationsverfahren durchgesetzt.

E Chirurgische Therapien bei OSA bieten $100 \%$ ige Therapieadhärenz.

\section{Frage 2}

Welches ist keine mögliche Folge der OSA?
A erhöhtes Risiko für Hypertonie
B erhöhtes Risiko für Schlaganfälle
C erhöhtes Risiko für Polyneuropathien
D erhöhtes Unfallrisiko im Straßenverkehr
E erhöhtes Risiko für Herzinsuffizienz

\section{Frage 3}

Welche Aussage trifft zu? Die Schlafendoskopie...

A ...kann die OSA erfolgreich behandeln.

B ...kann den Kollapsort, das Kollapsmuster und den Kollapsgrad der OSA bestimmen.

C ...wird in Allgemeinanästhesie durchgeführt.

D ...ist ein invasives Verfahren zur Therapie der OSA.

E ...muss stationär erfolgen.

\section{Frage 4}

Welche Aussage ist richtig? Die Stimulation des N. hypoglossus...

A ...stellt die primäre Therapie bei schwerer OSA dar.

B ...ist ein minimal- invasives Verfahren.

C ...stellt den Goldstandard zur Therapie der postoperativen Hypoglossusparese dar.

D ...kann bei mittel- bis schwergradiger OSA und Ineffektivität/ Unverträglichkeit der CPAP-Terapie empfohlen werden.

E ...basiert auf der bilateralen Stimulation des N. hypoglossus.

\section{Frage 5}

Welche Aussage ist falsch?

A Die Tracheotomie ist eine erfolgreiche chirurgische Therapieoption bei schwerer OSA.

B Die MMO ist eine erfolgreiche chirurgische Therapie bei schwerer OSA.

C Die UPPP inklusive Tonsillektomie ist eine minimal- invasive Behandlungsoption und kann in Lokalanästhesie durchgeführt werden.

D Die UPPP kann zu Komplikationen wie Nasopharynxstenose und velopharyngealer Insuffizienz führen.

E Die MMO ist technisch anspruchsvoll und gehört zu den invasiven Eingriffen zur Therapie der OSA.

\section{Frage 6}

Welche chirurgische Therapieoption gehört zu den minimal-invasiven Methoden?

A Interstitielle Radiofrequenztherapie und Weichgaumenimplantate

B Uvulopalatopharyngoplastik (UPPP)

C Hypoglossusnervstimulation

D CPAP-Beatmung

E Hyoidsuspension

\section{Frage 7}

Welches Verfahren zählt nicht zu den chirurgischen Verfahren der Therapie bei Rhonchopathie/OSA?

A Uvulopalatopharyngoplastik

B Laser-assistierte Uvulopalatoplastik

C interstitielle Radiofrequenztherapie des Weichgaumens und des Zungengrundes

D Laryngektomie

E Zungengrundreduktion

- Weitere Fragen auf der folgenden Seite ... 


\section{Punkte sammeln auf CME.thieme.de}

Fortsetzung $\ldots$

\section{Frage 8}

Was stellt keine Komplikation der OSA-Chirurgie dar?
A Ulzera
B velopharyngeale Insuffizienz
C Odynophagie
D Einblutung
E Paukenerguss

\section{Frage 9}

Welche anatomischen Strukturen werden in der VOTE-Einstellung nicht bewertet?
A Velum
B Epiglottis
C Nasenseptum
D Oropharynx
E Zungengrund

\section{Frage 10}

Welche Aussage trifft zu?

A Operativer Erfolg einer OSA-Therapie wird gemäß Sher et al. als Reduktion des AHI um $100 \%$ und unter einen Wert von 5 definiert.

B Operativer Erfolg einer OSA-Therapie wird gemäß Sher et al. als Reduktion des AHI um ein Drittel und unter einen Wert von 20 definiert.

C Operativer Erfolg einer OSA-Therapie wird gemäß Sher et al. als Reduktion des AHI um die Hälfte und über einen Wert von 20 definiert.

D Operativer Erfolg einer OSA-Therapie wird gemäß Sher et al. als Reduktion des $\mathrm{AHI}$ um die Hälfte und unter einen Wert von 20 definiert.

E Für den operativen Erfolg einer OSA-Therapie gibt es keine Empfehlungen und wird rein klinisch bestimmt. 